\title{
Charged rotating dilaton black branes in AdS universe
}

\author{
A. Sheykhi ${ }^{1,2 *}$ and S.H. Hendi ${ }^{3 \dagger}$ \\ ${ }^{1}$ Department of Physics, Shahid Bahonar University, P.O. Box 76175, Kerman, Iran \\ ${ }^{2}$ Research Institute for Astrophysics and Astronomy of Maragha (RIAAM), Maragha, Iran \\ 3 Department of Physics, College of Science, \\ Yasouj University, Yasouj 75914, Iran
}

\begin{abstract}
We present the metric for the $(n+1)$-dimensional charged rotating dilaton black branes with cylindrical or toroidal horizons in the background of anti-de Sitter spacetime. We find the suitable counterterm which removes the divergences of the action in the presence of the dilaton potential in all higher dimensions. We plot the Penrose diagrams of the spacetime and reveal that the spacetime geometry crucially modifies in the presence of the dilaton field. The conserved and thermodynamic quantities of the black branes are also computed.
\end{abstract}

\section{INTRODUCTION}

The motivation idea for studying higher dimensional black holes with a negative cosmological constant arises from the correspondence between the gravitating fields in an anti-de Sitter (AdS) spacetime and conformal field theory living on the boundary of the AdS spacetime [1]. It was argued that the thermodynamics of black holes in AdS spaces can be identified with that of a certain dual CFT in the high temperature limit [2]. Having the AdS/CFT correspondence idea at hand, one can gain some insights into thermodynamic properties and phase structures of strong 't Hooft coupling CFTs by studying thermodynamics of AdS black holes. According to the AdS/CFT correspondence, the rotating black holes in AdS space are dual to certain CFTs in a rotating space [3], while charged ones are dual to CFTs with chemical potential [4, 5]. The most general higher dimensional uncharged rotating black holes in AdS space have been recently found [3, 6]. As far as we know, rotating black holes for the Maxwell field minimally coupled to Einstein gravity in

\footnotetext{
*sheykhi@mail.uk.ac.ir

$\dagger$ hendi@mail.yu.ac.ir
} 
higher dimensions, do not exist in a closed form and one has to rely on perturbative or numerical methods to construct them in the background of asymptotically flat [7, 8] and AdS [9] spacetimes. There has also been recent interest in constructing the analogous charged rotating solutions in the framework of gauged supergravity in various dimensions [10 12].

There has been a renewed interest in studying scalar coupled solutions of general relativity ever since new black hole solutions have been found in the context of string theory. The low energy effective action of string theory contains two massless scalars namely dilaton and axion. The dilaton field couples in a nontrivial way to other fields such as gauge fields and results into interesting solutions for the background spacetime [13-16]. These solutions [1316], however, are all asymptotically flat. The presence of Liouville-type dilaton potential, which is regarded as the generalization of the cosmological constant, changes the asymptotic behavior of the solutions to be neither asymptotically flat nor (A)dS. While in the presence of one or two Liouville-type potential, black holes/branes which are neither asymptotically flat nor (A)dS have been explored in [17-26], magnetic dilaton solutions coupled to nonlinear electrodynamics have also been investigated [27]. Although these kind of solutions may shed some light on the possible extensions of AdS/CFT correspondence, they are physically less interesting due to their unusual asymptotic behavior. Recently, the dilaton potential leading to (A)dS-like solutions of dilaton gravity has been found [28 30]. It was shown that the cosmological constant is coupled to the dilaton in a very nontrivial way. With an appropriate combination of three Liouville-type dilaton potentials, a class of static dilaton black hole solutions in (A)dS spaces has been obtained by using a coordinates transformation which recast the solution in the schwarzschild coordinates system [28, 29]. Such potential may arise from the compactification of a higher dimensional supergravity model [31] which originates from the low energy limit of a background string theory. More recently, one of us has constructed a class of magnetic rotating solutions in four-dimensional Einstein-Maxwell-dilaton gravity with Liouville-type potential in the background of AdS spaces [32]. Although these solutions are not black holes and represent spacetimes with conic singularities, asymptotically AdS charged rotating black string solutions in four-dimensional Einstein-Maxwell-dilaton gravity has also been constructed [33]. So far, exact higher dimensional charged rotating dilaton black hole/brane solutions for an arbitrary dilaton-electromagnetic coupling constant in the background of AdS spacetime have not been constructed. In this paper we intend to construct exact, charged rotating dilaton black branes with cylindrical or toroidal horizons in 
higher dimensional AdS spacetimes and investigate their properties.

This paper is outlined as follows. Section II is devoted to a brief review of the field equations and the general formalism of calculating the conserved quantities. We shall also present the suitable counterterm which removes the divergences of the action in the presence of the dilaton potential. In section III, we construct the $(n+1)$-dimensional charged rotating dilaton black branes with a complete set of rotation parameters and investigate their properties. We also obtain the conserved and thermodynamical quantities of the $(n+1)$-dimensional black brane solutions. We finish with conclusion in the last section.

\section{BASIC EQUATIONS AND COUNTERTERM METHOD}

We consider the $(n+1)$-dimensional theory in which gravity is coupled to dilaton and Maxwell field with an action

$$
\begin{aligned}
I_{G}= & -\frac{1}{16 \pi} \int_{\mathcal{M}} d^{n+1} x \sqrt{-g}\left(R-\frac{4}{n-1}(\nabla \Phi)^{2}-V(\Phi)-e^{-4 \alpha \Phi /(n-1)} F_{\mu \nu} F^{\mu \nu}\right) \\
& -\frac{1}{8 \pi} \int_{\partial \mathcal{M}} d^{n} x \sqrt{-\gamma} \Theta(\gamma),
\end{aligned}
$$

where $R$ is the scalar curvature, $\Phi$ is the dilaton field, $F_{\mu \nu}=\partial_{\mu} A_{\nu}-\partial_{\nu} A_{\mu}$ is the electromagnetic field tensor, and $A_{\mu}$ is the electromagnetic potential. $\alpha$ is an arbitrary constant governing the strength of the coupling between the dilaton and the Maxwell field. The last term in Eq. (1) is the Gibbons-Hawking surface term. It is required for the variational principle to be well-defined. The factor $\Theta$ represents the trace of the extrinsic curvature for the boundary $\partial \mathcal{M}$ and $\gamma$ is the induced metric on the boundary. While $\alpha=0$ corresponds to the usual Einstein-Maxwell-scalar theory, $\alpha=1$ indicates the dilaton-electromagnetic coupling that appears in the low energy string action in Einstein's frame. For arbitrary value of $\alpha$ in (A)dS spaces the form of the dilaton potential in arbitrary dimensions is chosen as [29]

$$
\begin{aligned}
V(\Phi)= & \frac{2 \Lambda}{n\left(n-2+\alpha^{2}\right)^{2}}\left\{-\alpha^{2}\left[(n+1)^{2}-(n+1) \alpha^{2}-6(n+1)+\alpha^{2}+9\right] e^{-4(n-2) \Phi /[(n-1) \alpha]}\right. \\
& \left.+(n-2)^{2}\left(n-\alpha^{2}\right) e^{4 \alpha \Phi /(n-1)}+4 \alpha^{2}(n-1)(n-2) e^{-2 \Phi\left(n-2-\alpha^{2}\right) /[(n-1) \alpha]}\right\} .
\end{aligned}
$$

Here $\Lambda$ is the cosmological constant. For later convenience we redefine $\Lambda=-n(n-1) / 2 l^{2}$, where $l$ is the AdS radius of spacetime. It is clear the cosmological constant is coupled to the dilaton in a very nontrivial way. This type of the dilaton potential was introduced for the 
first time by Gao and Zhang [29]. They derived, by applying a coordinates transformation which recast the solution in the Schwarzchild coordinates system, the static dilaton black hole solutions in the background of (A)dS universe. For this purpose, they required the existence of the (A)dS dilaton black hole solutions and extracted successfully the form of the dilaton potential leading to (A)dS-like solutions. They also argued that this type of derived potential can be obtained when a higher dimensional theory is compactified to four dimension, including various supergravity models [31]. In the absence of the dilaton field $(\Phi=0=\alpha)$, the potential (2) reduces to $V(\Phi)=2 \Lambda$, and the action (11) recovers the action of Einstein-Maxwell gravity with cosmological constant. The equations of motion can be obtained by varying the action (11) with respect to the gravitational field $g_{\mu \nu}$, the dilaton field $\Phi$ and the gauge field $A_{\mu}$ which yields the following field equations

$$
\begin{gathered}
\mathcal{R}_{\mu \nu}=\frac{4}{n-1}\left(\partial_{\mu} \Phi \partial_{\nu} \Phi+\frac{1}{4} g_{\mu \nu} V(\Phi)\right)+2 e^{-4 \alpha \Phi /(n-1)}\left(F_{\mu \eta} F_{\nu}^{\eta}-\frac{1}{2(n-1)} g_{\mu \nu} F_{\lambda \eta} F^{\lambda \eta}\right) \\
\nabla^{2} \Phi=\frac{n-1}{8} \frac{\partial V}{\partial \Phi}-\frac{\alpha}{2} e^{-4 \alpha \Phi /(n-1)} F_{\lambda \eta} F^{\lambda \eta} \\
\partial_{\mu}\left(\sqrt{-g} e^{-4 \alpha \Phi /(n-1)} F^{\mu \nu}\right)=0 .
\end{gathered}
$$

The conserved charges of the spacetime can be calculated through the use of the substraction method of Brown and York [34]. Such a procedure causes the resulting physical quantities to depend on the choice of reference background. For asymptotically AdS solutions, the way that one can calculate these quantities and obtain finite values for them is through the use of the counterterm method inspired by AdS/CFT correspondence [1]. In this paper we deal with the spacetimes with zero curvature boundary, $R_{a b c d}(\gamma)=0$, and therefore the counterterm for the stress energy tensor should be proportional to $\gamma^{a b}$. We find the suitable counterterm which removes the divergences in the form

$$
I_{c t}=-\frac{1}{8 \pi} \int_{\partial \mathcal{M}} d^{n} x \sqrt{-\gamma}\left(-\frac{(n-1)(n-2)}{2 l}+\frac{\sqrt{-n(n-1) V(\Phi)}}{2}\right) .
$$

In the absence of the dilaton field, $V(\Phi)=2 \Lambda=-n(n-1) / l^{2}$, and Eq. (6) reduces to

$$
I_{c t}=-\frac{1}{8 \pi} \int_{\partial \mathcal{M}} d^{n} x \sqrt{-\gamma}\left(\frac{n-1}{l}\right)
$$

which is the counterterm of the asymptotically AdS spaces. Having the total finite action $I=I_{G}+I_{\text {ct }}$ at hand, we can use the quasilocal definition to construct a divergence free 
stress-energy tensor [34]. Thus we write down the finite stress-energy tensor in $(n+1)$ dimensional Einstein-dilaton gravity with three Liouville-type dilaton potentials (2) in the following form

$$
T^{a b}=\frac{1}{8 \pi}\left[\Theta^{a b}-\Theta \gamma^{a b}+\left(-\frac{(n-1)(n-2)}{2 l}+\frac{\sqrt{-n(n-1) V(\Phi)}}{2}\right) \gamma^{a b}\right] .
$$

The first two terms in Eq. (8) are the variation of the action (11) with respect to $\gamma_{a b}$, and the last two terms are the variation of the boundary counterterm (6) with respect to $\gamma_{a b}$. To compute the conserved charges of the spacetime, one should choose a spacelike surface $\mathcal{B}$ in $\partial \mathcal{M}$ with metric $\sigma_{i j}$, and write the boundary metric in ADM (Arnowitt-Deser-Misner) form:

$$
\gamma_{a b} d x^{a} d x^{a}=-N^{2} d t^{2}+\sigma_{i j}\left(d \varphi^{i}+V^{i} d t\right)\left(d \varphi^{j}+V^{j} d t\right)
$$

where the coordinates $\varphi^{i}$ are the angular variables parameterizing the hypersurface of constant $r$ around the origin, and $N$ and $V^{i}$ are the lapse and shift functions respectively. When there is a Killing vector field $\xi$ on the boundary, then the quasilocal conserved quantities associated with the stress tensors of Eq. (8) can be written as

$$
Q(\xi)=\int_{\mathcal{B}} d^{n-1} x \sqrt{\sigma} T_{a b} n^{a} \xi^{b},
$$

where $\sigma$ is the determinant of the metric $\sigma_{i j}, \xi$ and $n^{a}$ are, respectively, the Killing vector field and the unit normal vector on the boundary $\mathcal{B}$. For boundaries with timelike $(\xi=\partial / \partial t)$ and rotational $(\varsigma=\partial / \partial \varphi)$ Killing vector fields, one obtains the quasilocal mass and angular momentum

$$
\begin{aligned}
M & =\int_{\mathcal{B}} d^{n-1} x \sqrt{\sigma} T_{a b} n^{a} \xi^{b}, \\
J & =\int_{\mathcal{B}} d^{n-1} x \sqrt{\sigma} T_{a b} n^{a} \varsigma^{b} .
\end{aligned}
$$

provided the surface $\mathcal{B}$ contains the orbits of $\varsigma$. These quantities are, respectively, the conserved mass and angular momenta of the system enclosed by the boundary $\mathcal{B}$. Note that they will both depend on the location of the boundary $\mathcal{B}$ in the spacetime, although each is independent of the particular choice of foliation $\mathcal{B}$ within the surface $\partial \mathcal{M}$.

\section{CHARGED ROTATING DILATON BLACK BRANE}

Our aim here is to construct the $(n+1)$-dimensional rotating solutions of the field equations (3)-(5) with $k$ rotation parameters and investigate their properties. The rotation group 
in $(n+1)$-dimensions is $S O(n)$ and therefore the number of independent rotation parameters for a localized object is equal to the number of Casimir operators, which is $[n / 2] \equiv k$, where $[x]$ is the integer part of $x$. Inspired by [35], we take the metric of $(n+1)$-dimensional rotating solution with cylindrical or toroidal horizons and $k$ rotation parameters in the form

$$
\begin{aligned}
d s^{2}= & -U(r)\left(\Xi d t-\sum_{i=1}^{k} a_{i} d \phi_{i}\right)^{2}+\frac{r^{2}}{l^{4}} R^{2}(r) \sum_{i=1}^{k}\left(a_{i} d t-\Xi l^{2} d \phi_{i}\right)^{2} \\
& -\frac{r^{2}}{l^{2}} R^{2}(r) \sum_{i<j}^{k}\left(a_{i} d \phi_{j}-a_{j} d \phi_{i}\right)^{2}+\frac{d r^{2}}{W(r)}+\frac{r^{2}}{l^{2}} R^{2}(r) d X^{2}, \\
\Xi^{2}= & 1+\sum_{i=1}^{k} \frac{a_{i}^{2}}{l^{2}}
\end{aligned}
$$

where $a_{i}$ 's are $k$ rotation parameters. The functions $U(r), W(r)$ and $R(r)$ should be determined and $l$ has the dimension of length which is related to the cosmological constant $\Lambda$ for the case of Liouville-type potential with constant $\Phi$. The angular coordinates are in the range $0 \leq \phi_{i} \leq 2 \pi$ and $d X^{2}$ is the Euclidean metric on the $(n-k-1)$-dimensional submanifold with volume $\Sigma_{n-k-1}$. The Maxwell equation (5) can be integrated immediately to give

$$
\begin{aligned}
F_{t r} & =\sqrt{\frac{U(r)}{W(r)}} \frac{q \Xi e^{4 \alpha \Phi /(n-1)}}{(r R)^{n-1}}, \\
F_{\phi_{i} r} & =-\frac{a_{i}}{\Xi} F_{t r} .
\end{aligned}
$$

where $q$, an integration constant, is the charge parameter of the black brane. Using metric (12) and the Maxwell fields (13), one can show that equations (3)-(44) have solutions of the form

$$
\begin{aligned}
& U(r)=-\left(\frac{c}{r}\right)^{n-2}\left[1-\left(\frac{b}{r}\right)^{n-2}\right]^{1-\gamma(n-2)}-\frac{2 \Lambda r^{2}}{n(n-1)}\left[1-\left(\frac{b}{r}\right)^{n-2}\right]^{\gamma}, \\
& W(r)=\left\{-\left(\frac{c}{r}\right)^{n-2}\left[1-\left(\frac{b}{r}\right)^{n-2}\right]^{1-\gamma(n-2)}-\frac{2 \Lambda r^{2}}{n(n-1)}\left[1-\left(\frac{b}{r}\right)^{n-2}\right]^{\gamma}\right\} \\
& \times\left[1-\left(\frac{b}{r}\right)^{n-2}\right]^{\gamma(n-3)}, \\
& \Phi(r)=\frac{n-1}{4} \sqrt{\gamma(2+2 \gamma-n \gamma)} \ln \left[1-\left(\frac{b}{r}\right)^{n-2}\right], \\
& R(r)=\left[1-\left(\frac{b}{r}\right)^{n-2}\right]^{\gamma / 2},
\end{aligned}
$$


Here $b$ and $c$ are integration constants and the constant $\gamma$ is

$$
\gamma=\frac{2 \alpha^{2}}{(n-2)\left(n-2+\alpha^{2}\right)}
$$

The charge parameter $q$ is related to $b$ and $c$ by

$$
q^{2}=\frac{(n-1)(n-2)^{2}}{2\left(n-2+\alpha^{2}\right)} b^{n-2} c^{n-2} .
$$

When $(\alpha=0=\gamma)$, the above solution recovers the asymptotically AdS charged rotating black branes presented in [35, 36]. In the particular case $n=3$ these solutions reduce to the asymptotically AdS charged rotating dilaton black strings [33]. For $n=3$ and $\alpha=0$ they reduce to charged rotating black string solutions presented in [37]. Inserting solutions (14)-(17) into the Maxwell fields (13), they can be simplified as

$$
\begin{aligned}
F_{t r} & =\frac{q \Xi}{r^{n-1}}, \\
F_{\phi_{i} r} & =-\frac{a_{i}}{\Xi} F_{t r} .
\end{aligned}
$$

As one can see from Eq. (20), in the background of AdS universe, the dilaton field does not exert any direct influence on the matter fields $F_{t r}$ and $F_{\phi_{i} r}$ 's $(i=1, \ldots, k)$, however, the dilaton field modifies the geometry of the spacetime as it participates in the field equations. This is in contrast to the solutions presented in [23]. The solutions of Ref. [23] are neither asymptotically flat nor (A)dS and the gauge field crucially depends on the scalar dilaton field. The gauge potential $A_{\mu}$ corresponding to the electromagnetic tensor (20) can be obtained as

$$
A_{\mu}=-\frac{q}{(n-2) r^{n-2}}\left(\Xi \delta_{\mu}^{t}-a_{i} \delta_{\mu}^{i}\right) \quad \text { (no sum on i). }
$$

This spacetime is asymptotically AdS, since the functions $W(r)$ and $U(r)$ behave as $-2 \Lambda\left(r^{2} /[n(n-1)]\right)$ as $r \rightarrow \infty$. Indeed, for large values of $r$, in four dimensions $(n=3)$ the functions $W(r)$ and $U(r)$ behave as $-2 \Lambda\left(r^{2} / 6+p_{1} r+p_{2}\right)$, while in higher dimensions $(n \geq 4)$ they behave like $-2 \Lambda r^{2} /[n(n-1)]$. Here $p_{1}$ and $p_{2}$ are functions of $\alpha$. This implies that the falloff rate of the solutions in four dimension is much slower than in higher dimensions. The Kretschmann invariant $R_{\mu \nu \lambda \kappa} R^{\mu \nu \lambda \kappa}$ and the Ricci scalar $R$ diverge at $r=0$ and therefore there is an essential singularity located at $r=0$. For all $\alpha$, the surface $r=r_{+}$ is an event horizon (the positive root of Eq. $W\left(r=r_{+}\right)=0$ ). The surface $r=b$ is a curvature singularity except for the case $\alpha=0$ when it is a nonsingular inner horizon. This 


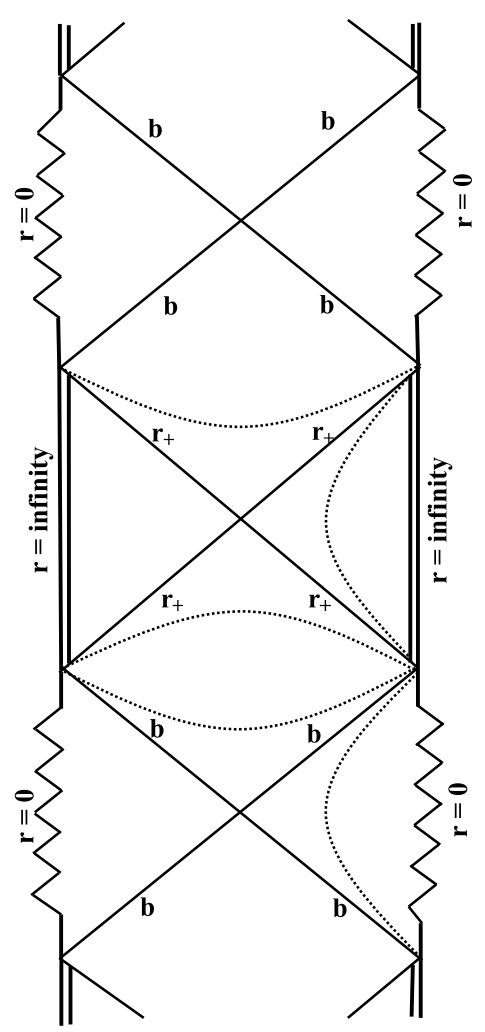

FIG. 1: Penrose diagram for black brane with two horizon $(\alpha=0)$ located at $r=b$ and $r=r_{+}$. The dotted curves represent $r=$ const.

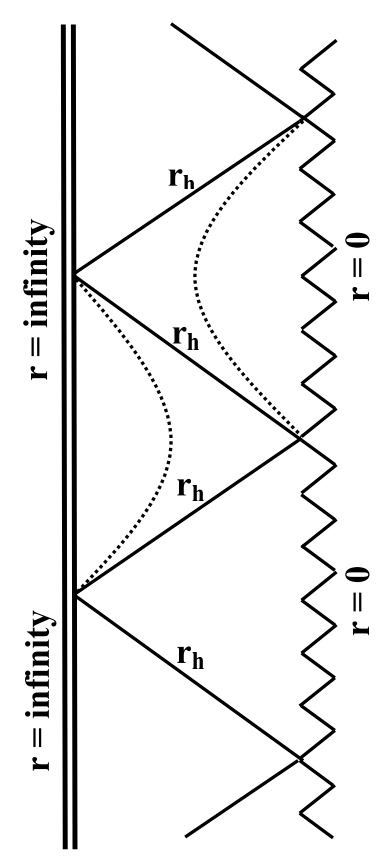

FIG. 2: Penrose diagram for extreme black brane with $\alpha=0$ and one horizon located at $r=r_{+}=$ $r_{\mathrm{h}}$. The dotted curves represent $r=$ const. 


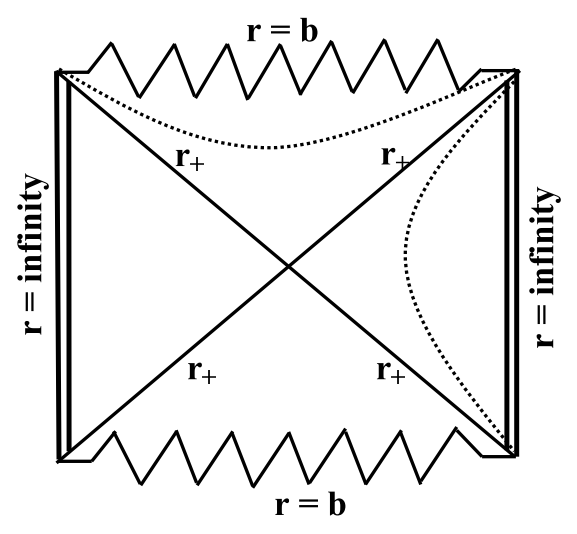

FIG. 3: Penrose diagram for black brane with $\alpha \neq 0$ and one horizon located at $r_{+}$. The dotted curves represent $r=$ const.

is consistent with the idea that the inner horizon is unstable in the Einstein-Maxwell theory. Therefore, our solutions describe black branes only in the case $b<r_{+}[16]$. For $\alpha=0$ the metric (12) is real in the range $0 \leq r<\infty$, while for $\alpha>0$, it is real only in the range $b \leq r<\infty$. Thus, in order to have a real metric, we restrict the spacetime to the region $r \geq b$. We plot Penrose diagrams of spacetime in Figs. 113. From these figures we find out that the casual structure is asymptotically well behaved. It is notable to mention that in contrast to the solutions of Ref. [25], here we have a spacelike singularity with one horizon and the solutions are asymptotically AdS, at all times, in the presence of dilaton field. It is also worthwhile to note that the dilaton field $\Phi(r)$ and the electromagnetic fields $F_{t r}$ and $F_{\phi_{i} r}$ 's become zero as $r \rightarrow \infty$. As in the case of rotating black hole solutions of the Einstein gravity, the above metric has both Killing and event horizons. The Killing horizon is a null surface whose null generators are tangent to a Killing field. It is easy to see that the Killing vector

$$
\chi=\partial_{t}+\sum_{i=1}^{k} \Omega_{i} \partial_{\phi_{i}}
$$

is the null generator of the event horizon, where $k$ denote the number of rotation parameters [38]. We can obtain the temperature and angular velocity of the horizon by analytic continuation of the metric. The analytical continuation of the Lorentzian metric by $t \rightarrow i \tau$ and $a \rightarrow i a$ yields the Euclidean section, whose regularity at $r=r_{+}$requires that we should identify $\tau \sim \tau+\beta_{+}$and $\phi_{i} \sim \phi_{i}+\beta_{+} \Omega_{i}$, where $\beta_{+}$and $\Omega_{i}$ 's are the inverse Hawking temperature and the $i$ th component of angular velocity of the horizon, respectively. The Hawking 
temperature of the black brane on the horizon $r_{+}$can be calculated using the relation

$$
T_{+}=\beta_{+}^{-1}=\left(\frac{U^{\prime}}{4 \pi \Xi \sqrt{U / W}}\right)_{r=r_{+}} .
$$

where a prime denotes derivative with respect to $r$. It is a matter of calculation to show that

$$
\begin{aligned}
T_{+} & =\frac{1}{4 \pi \Xi r_{+}}\left\{n+(n-1)[\gamma(n-2)-2]\left(\frac{b}{r_{+}}\right)^{n-2}\right\}\left(\frac{c}{r_{+}}\right)^{n-2}\left[1-\left(\frac{b}{r_{+}}\right)^{n-2}\right]^{-\gamma(n-1) / 2} \\
\Omega_{i} & =\frac{a_{i}}{\Xi l^{2}}
\end{aligned}
$$

where we have used equation $W\left(r=r_{+}\right)=0$ for omitting $\Lambda$. It is easy to check that for $\alpha \geq \sqrt{n}$, the temperature is always positive, while for $\alpha<\sqrt{n}$ the temperature is positive definite provided we have

$$
r_{+}>b\left(\frac{-n}{(n-1)[\gamma(n-2)-2]}\right)^{-1 /(n-2)}
$$

The entropy of the black brane typically satisfies the so called area law of the entropy which states that the entropy of the black hole is a quarter of the event horizon area [39]. This near universal law applies to almost all kinds of black holes, including dilaton black holes/branes, in Einstein gravity [40]. Denoting the volume of the hypersurface boundary at constant $t$ and $r$ by $V_{n-1}=(2 \pi)^{k} \Sigma_{n-k-1}$, we can show that the entropy per unit volume $V_{n-1}$ of the black brane is

$$
S=\frac{\Xi r_{+}^{n-1}}{4 l^{n-2}}\left[1-\left(\frac{b}{r_{+}}\right)^{n-2}\right]^{\gamma(n-1) / 2} .
$$

The mass per unit volume $V_{n-1}$ of the black brane can be calculated through the use of Eq. (10). We find

$$
\begin{array}{ll}
M=\frac{\left(3 \Xi^{2}-1\right) c}{16 \pi l}+\frac{\alpha^{2}\left(\alpha^{2}-1\right) b^{3}}{24 \pi l^{3}\left(\alpha^{2}+1\right)^{3}} & \text { for } n=3, \\
M=\frac{\left(n \Xi^{2}-1\right) c^{n-2}}{16 \pi l^{n-2}} & \text { for } n \geq 4 .
\end{array}
$$

Let us note that the mass expression in four dimension differs from higher dimensions. This is due to the fact that, for large values of $r$, the falloff rate of the solutions in four and higher dimensions are different. Thus, the mass in four dimension depends on the dilaton coupling constant $\alpha$ while in higher dimensions it is independent of $\alpha$ and coincides with the mass 
of charged rotating black branes in Einstein gravity [36]. The angular momentum per unit volume $V_{n-1}$ of the black brane can be calculated through the use of Eqs. (11). We obtain

$$
J_{i}=\frac{n \Xi c^{n-2} a_{i}}{16 \pi l^{n-2}}
$$

For $a_{i}=0(\Xi=1)$, the angular momentum per unit volume vanishes, and therefore $a_{i}$ 's are the rotational parameters of the spacetime. Next, we calculate the electric charge of the solutions. To determine the electric field we should consider the projections of the electromagnetic field tensor on special hypersurfaces. The normal to such hypersurfaces is

$$
u^{0}=\frac{1}{N}, \quad u^{r}=0, \quad u^{i}=-\frac{V^{i}}{N}
$$

where $N$ and $V^{i}$ are the lapse function and shift vector. Then the electric field is $E^{\mu}=$ $g^{\mu \rho} e^{\frac{-4 \alpha \phi}{n-1}} F_{\rho \nu} u^{\nu}$, and the electric charge per unit volume $V_{n-1}$ can be found by calculating the flux of the electric field at infinity, yielding

$$
Q=\frac{\Xi q}{4 \pi l^{n-2}}
$$

The electric potential $U$, measured at infinity with respect to the horizon, is defined by [5, 41]

$$
U=\left.A_{\mu} \chi^{\mu}\right|_{r \rightarrow \infty}-\left.A_{\mu} \chi^{\mu}\right|_{r=r_{+}}
$$

where $\chi$ is the null generators of the event horizon given by Eq. (22). It is a matter of calculation to show that

$$
U=\frac{q}{(n-2) \Xi r_{+}^{n-2}}
$$

\section{CONCLUSIONS}

It is well-known that in the presence of Liouville-type dilaton potential, which is regarded as the generalization of the cosmological constant, the asymptotic behavior of the solutions change to be neither asymptotically flat nor (A)dS. As a matter of fact, with the exception of a pure cosmological constant, no dilaton (A)dS solution exists with the presence of only one or two Liouville-type potential [17]. In this paper, with an appropriate combination of three Liouville-type dilaton potentials, we obtained a new class of charged rotating black brane solutions in $(n+1)$-dimensional Einstein-Maxwell-dilaton gravity with cylindrical or toroidal horizons in the background of AdS spaces and investigated their properties. We 
found a suitable counterterm which removes the divergences of the action in the presence of dilaton potential in all higher dimensions. We ploted the Penrose diagrams associated with these spacetimes. These diagrams show that for $\alpha=0$, the solutions can be interpreted as black brane with two event horizons, an extreme black brane or a naked singularity provided the parameters of the solutions are chosen suitably. In this case we encounter a timelike singularity which is located at $r=0$. We found out that for $\alpha>0$, the solutions represent black brane with one horizon. In this case we have a spacelike singularity at $r=b$. We also computed the conserved and thermodynamic quantities of the black branes by using the conterterm method inspired by AdS/CFT correspondence. Interestingly enough, we found that the mass expression in four dimension depends on the dilaton coupling constant $\alpha$, while in higher dimensions it is independent of $\alpha$. This can be understood easily, since in four dimension the functions $W(r)$ and $U(r)$ behave as $-2 \Lambda\left(r^{2} / 6+p_{1} r+p_{2}\right)$ for large $r$, while in higher dimensions $(n \geq 4)$ they behave like $-2 \Lambda r^{2} /[n(n-1)]$.

\section{Acknowledgments}

We thank the anonymous referee for constructive comments. We are also grateful to Prof. M.H. Dehghani for helpful discussions. This work has been supported by Research Institute for Astronomy and Astrophysics of Maragha.

[1] E. Witten, Adv. Theor. Math. Phys. 2, 253 (1998);

J. M. Maldacena, Adv. Theor. Math. Phys. 2, 231 (1998).

[2] E. Witten, Adv. Theor. Math. Phys. 2, 505 (1998).

[3] S. W. Hawking, C. J. Hunter and M. Taylor, Phys. Rev. D 59, 064005 (1999).

[4] A. Chamblin, R. Emparan, C. V. Johnson and R. C. Myers, Phys. Rev. D 60, 064018 (1999);

R. G. Cai and K. S. Soh, Mod. Phys. Lett. A 14, 1895 (1999).

[5] M. Cvetic and S. S. Gubser, J. High Energy Phys. 04, 024 (1999).

[6] G. W. Gibbons, H. Lu, D. N. Page and C. N. Pope, Phys. Rev. Lett. 93, 171102 (2004);

G. W. Gibbons, H. Lu, D. N. Page and C. N. Pope, J. Geom. Phys. 53, 49 (2005).

[7] J. Kunz, F. Navarro-Lerida and J. Viebahn, Phys. Lett. B 639, 362 (2006). 
[8] A. N. Aliev, Phys. Rev. D 74, 024011 (2006).

[9] J. Kunz, F. Navarro-Lerida and E. Radu, Phys. Lett. B 649, 463 (2007);

A. N. Aliev, Phys. Rev. D 75, 084041 (2007);

H. C. Kim and R. G. Cai, Phys. Rev. D 77, 024045 (2008);

Y. Brihaye and T. Delsate, arXiv:0806.1583.

[10] M. Cvetic and D. Youm, Phys. Rev. D 54, 2612 (1996);

D. Youm, Phys. Rep. 316, 1 (1999).

[11] M. Cvetic and D. Youm, Nucl. Phys. B 477, 449 (1996).

[12] M. Cvetic and D. Youm, Nucl. Phys. B 476, 118 (1996);

Z. W. Chong, M. Cvetic, H. Lu, and C. N. Pope, Phys. Rev. D 72, 041901 (2005).

[13] G. W. Gibbons and K. Maeda, Nucl. Phys. B 298, 741 (1988);

T. Koikawa and M. Yoshimura, Phys. Lett. B 189, 29 (1987);

D. Brill and J. Horowitz, Phys. Lett. B 262, 437 (1991).

[14] D. Garfinkle, G. T. Horowitz and A. Strominger, Phys. Rev. D 43, 3140 (1991);

R. Gregory and J. A. Harvey, Phys. Rev. D 47, 2411 (1993).

[15] G. T. Horowitz and A. Strominger, Nucl. Phys. B 360, 197 (1991).

[16] J. H. Horn and G. Horowitz, Phys. Rev. D 46, 1340 (1992).

[17] S. J. Poletti and D. L. Wiltshire, Phys. Rev. D 50, 7260 (1994) ;

S. J. Poletti, J. Twamley and D. L. Wiltshire, Phys. Rev. D 51, 5720 (1995).

[18] K. C. K. Chan, J. H. Horne and R. B. Mann, Nucl. Phys. B 447, 441 (1995).

[19] R. G. Cai, J. Y. Ji and K. S. Soh, Phys. Rev D 57, 6547 (1998);

R. G. Cai and Y. Z. Zhang, ibid. 64, 104015 (2001).

[20] G. Clement, D. Gal'tsov and C. Leygnac, Phys. Rev D 67, 024012 (2003);

G. Clement and C. Leygnac, Phys. Rev. D 70, 084018 (2004).

[21] M. H Dehghani, Phys. Rev. D 71, 064010 (2005).

[22] M. H. Dehghani and N. Farhangkhah, Phys. Rev. D 71, 044008 (2005).

[23] A. Sheykhi, M. H. Dehghani and N. Riazi, Phys. Rev. D 75, 044020 (2007);

A. Sheykhi, M. H. Dehghani, N. Riazi and J. Pakravan Phys. Rev. D 74, 084016 (2006);

A. Sheykhi and N. Riazi, Phys. Rev. D 75, 024021 (2007);

R.G. Cai, Y.Z. Zhang, Phys. Rev. D 544891 (1996).

[24] A. Sheykhi, Phys. Rev. D 76, 124025 (2007); 
A. Sheykhi, Phys. Lett. B 662, 7 (2008).

[25] M. H. Dehghani, J. Pakravan and S. H. Hendi, Phys. Rev. D 74, 104014 (2006);

S. H. Hendi, J. Math. Phys. 49, 082501 (2008).

[26] M. H. Dehghani, S. H. Hendi, A. Sheykhi and H. Rastegar Sedehi. JCAP 0702, 020 (2007).

[27] M. H. Dehghani, A. Sheykhi and S. H. Hendi, Phys. Lett. B 659, 476 (2008).

[28] C. J. Gao and S. N. Zhang, Phys. Rev. D 70, 124019 (2004).

[29] C. J. Gao and S. N. Zhang, Phys. Lett. B 605, 185 (2005).

[30] C. J. Gao and S. N. Zhang, Phys. Lett. B 612127 (2005).

[31] S. B. Giddings, Phys. Rev. D 68, 026006 (2003);

E. Radu and D. H. Tchrakian, Class. Quant. Grav. 22, 879 (2005).

[32] A. Sheykhi, Phys. Lett. B 672,101 (2009).

[33] A. Sheykhi, Phys. Rev. D 78, 064055 (2008).

[34] J. Brown and J. York, Phys. Rev. D 47, 1407 (1993).

[35] A. M. Awad, Class. Quant. Grav. 20, 2827 (2003).

[36] M. H. Dehghani, Phys. Rev. D 66, 044006 (2002);

M. H. Dehghani and A. Khodam-Mohammadi, ibid. 67, 084006 (2003).

[37] J. P.S. Lemos and V. T. Zanchin, Phys. Rev. D 54, 3840 (1996);

J. P. S. Lemos, Class. Quant. Grav. 12, 1081 (1995);

J. P. S. Lemos, Phys. Lett. B 353, 46 (1995).

[38] M. H. Dehghani and R. B. Mann, Phys.Rev. D 73, 104003 (2006).

[39] J. D. Beckenstein, Phys. Rev. D 7, 2333 (1973);

S. W. Hawking, Nature (London) 248, 30 (1974);

G. W. Gibbons and S. W. Hawking, Phys. Rev. D 15, 2738 (1977).

[40] C. J. Hunter, Phys. Rev. D 59, 024009 (1999);

S. W. Hawking, C. J Hunter and D. N. Page, Phys. Rev. D 59, 044033 (1999);

R. B. Mann Phys. Rev. D 60, 104047 (1999).

[41] M. M. Caldarelli, G. Cognola and D. Klemm, Class. Quant. Grav. 17, 399 (2000). 